\title{
Kinetic resolution of a $\beta$-adrenolytic drug with the use of lipases as enantioselective biocatalysts
}

\author{
Corresponding author: \\ Prof. Michał Piotr Marszałt \\ Collegium Medicum in Bydgoszcz \\ Jurasza 2 St. \\ 85-089 Bydgoszcz \\ Poland \\ Tel.: +48525853540 \\ Fax: +48525853529 \\ e-mail address: mmars@cm.umk.pl \\ Medical Research Journal 2017; \\ Volume 2, Number 4, 38-42 \\ 10.5603/MRJ.2017.0007 \\ Copyright (C) 2017 Via Medica \\ ISSN 2451-2591
}

\begin{abstract}
The study presented herein is focused on optimisation of the kinetic resolution of racemic propranolol by screening acetylating agent, reaction medium, and enantioselective biocatalysts. The effects of acetylating agent and reaction medium on the efficiency of performed enantioselective biotransformation were investigated. Furthermore, the catalytic activities on nine commercially available lipases were tested. Finally, the composition of reaction medium was selected, based on previously obtained results, providing the most efficient process of kinetic resolution of racemic propranolol.

Key words: enzyme, $(R, S)$-propranolol, kinetic resolution, enantioselective acetylation, Candida rugosa (OF) lipase.
\end{abstract}

Med Res J 2018; 3 (1): 38-42

\section{Introduction}

Cardiovascular diseases and heart diseases, also known as cardiological diseases, are the most common cause of death in developed countries, including Poland. Additionally, cardiovascular diseases are the most widespread group of diseases not only among the elderly. The causes of cardiovascular problems are diverse, which is why medicines used to fight them belong to many groups. The most common cardiological diseases are: arterial hypertension, ischaemic heart disease, arrhythmias, or heart failure. The treatment strategies of these diseases consist of the use of antihypertensive drugs, i.e. those that lower blood pressure, drugs that improve heart function, operate antiarrhythmics, or change blood properties (1).

Propranolol belongs to the $\beta$-adrenergic receptor antagonists ( $\beta$-blockers) class, which make up an important class of drugs widely used in the treatment of hypertension and cardiovascular disorders (2-5). Although propranolol is one of the oldest non-selective beta receptor blocking agents, it has well known physical and chemical properties. Hence it can be use in a comparative study of the catalytic activity of different enzymes in biocatalysis reactions. Because $\beta$-blockers have an asymmetric carbon atom in their structure, they occur in two enantiomeric forms. Although the two enantiomers of $\beta$-blockers feature the same chemical and physical properties in an achiral environment, they differ in pharmacological activity. It has been reported by many studies that only the $S$-enantiomer of $\beta$-blockers has the desired therapeutic effect, whereas the administration of the racemate may cause dangerous side effects, such as bronchoconstriction or diabetes (6-9). Nevertheless, $\beta$-blockers are still commercially available and mainly used in medicine as racemates.

The main aim of the study herein was to obtain enantiomerically pure (S)-propranolol acetate, which is responsible for the therapeutic effect. Additionally, enzyme screening and reaction medium selection were performed to select the best reaction conditions. The chiral compounds obtained as a result of enantioselective acetylation of $(R, S)$-propranolol were analysed using chiral stationary phases and UPLC-MS/MS system. Moreover, to optimise the chiral chromatographic conditions, the type of stationary phase and mobile phase composition of the chromatographic process were selected 


\section{Materials and methods}

\section{Chemicals}

$(R, S)$-propranolol, $(R)$-propranolol, Burholderia cepacia lipase, Candida antarctica lipase, Candida rugosa lipase, toluene, $n$-hexane, isopropenyl acetate, vinyl acetate,

2-propanol, acetonitrile, diethylamine, and acetyl chloride were purchased from Sigma-Aldrich Co. (Stainhaim, Germany). Anhydrous sodium sulphate, sodium sulphate decahydrate, $4 \AA$ molecular sieves, and ethanol were purchased from POCH S.A. (Gliwice, Poland). Lipases OF and MY from Candida rugosa, lipase QLM from Alcaigenes fecalis, lipase PL from Alcaigenes sp., lipase TL from Pseudomonas stutzeri, and lipase SL from Burkholderia cepacia were a gift from Meito Sangyo Co. Ltd. (Japan). The water used in the study was obtained using a Milli-Q Water Purification System (Millipore, Bedford, MA, USA). All incubations were performed at controlled temperature and number of rotations (250 rpm). Glassware was oven dried for several hours, assembled hot, and cooled in a stream of nitrogen.

\section{Instrumentation}

The Refrigerated CentriVap Concentrator was purchased from Labconco; the Inkubator +1000 and Unimax 1010 were purchased from Heidolph; and the Shimadzu UPLC-MS/MS system (Japan) was equipped with two LC-30AD solvent delivery pumps combined with gradient systems, a degasser model DGU-20A5, an autosampler model SIL-30AC, a col- umn oven model CTO-20AC, and a UV detector model SPD-M20A. A Lux Celullose-1 (LC-1) column with a cellulose tris(3,5-dimetylphenylcarbamate) stationary phase, a Lux Cellulose-2 (LC-2) column with a cellulose tris(3-chloro-4methylphenylcarbamate) stationary phase, a Lux Cellulose-3 (LC-3) column with a cellulose tris(4-methylbenzoate) stationary phase, a Lux Amulose-2 (LA-2) column with an amylose tris(5-chloro-2-methylphenylcarbamate) stationary phase, and a Guard Cartridge System model KJO-4282 were purchased from Phenomenex Co.

\section{Chromatographic conditions}

To achieve satisfactory chromatographic resolution of $(R, S)$-propranolol and its esters, the effects of both the composition, flow rate of the mobile phase, and the type of chiral stationary phase (CSP) were investigated. Five types of CSPs were tested in normal, reversed, and polar/organic phase. Finally, the Lux Cellulose-1 column was chosen as an optimal stationary phase for chromatographic separation in the polar/organic phase mode (Figure 1). The mobile phase consisted of methanol/isopropanol/diethylamine/formic acid in a ratio of 99.8/0.2/0.1/0.1 (v/v/v/v). The chromatographic process was performed at $25^{\circ} \mathrm{C}$. The detection was performed with the use of mass spectrometry in MRM mode. To determine the optical purity and enantioselectivity of the enantioselective acetylation, previously used equations based on peak areas from a chromatogram obtained by the chromatographic separation of $(R, S)$-propranolol and its derivatives were applied $(10,11)$.

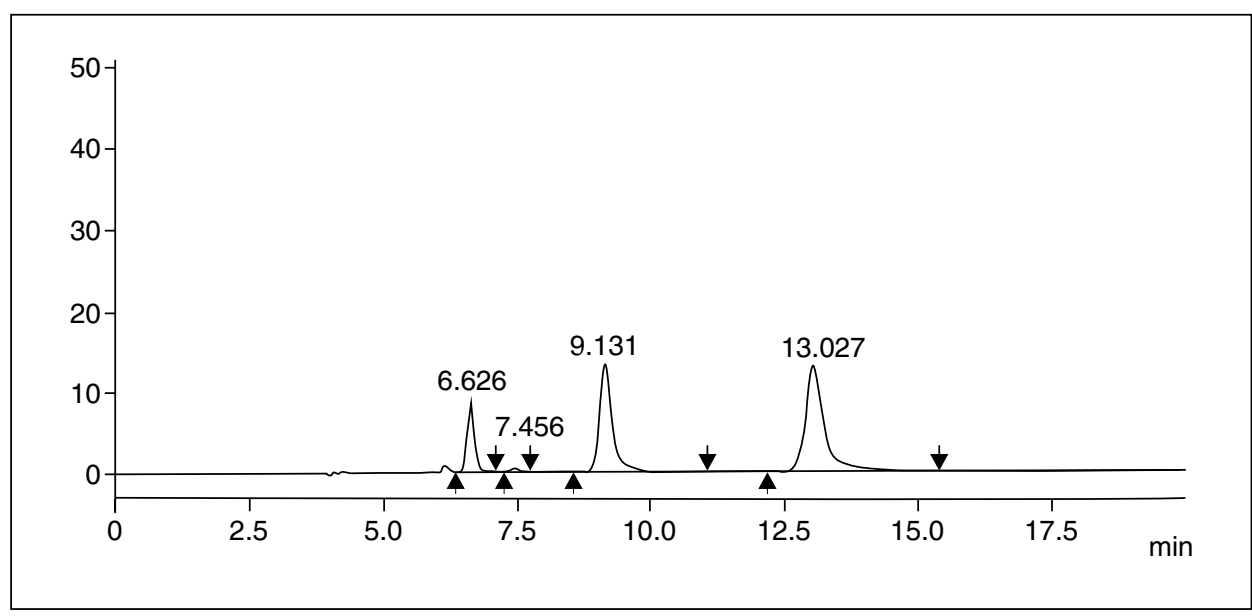

Figure 1. Chromatogram of racemic propranolol and its esters after enantioselective biotransformation of $(R, S)$-propranolol with the use of Candida rugosa lipase OF: $(S)$-enantiomer of propranolol acetate $\left(t_{\mathrm{R}}=6.626\right)$, $(R)$-enantiomer of propranolol acetate $\left(t_{R}=7.456\right),(S)$-propranolol $\left(t_{R}=9.131\right)$, and $(R)$-propranolol $\left(t_{R}=13.027\right)$. 


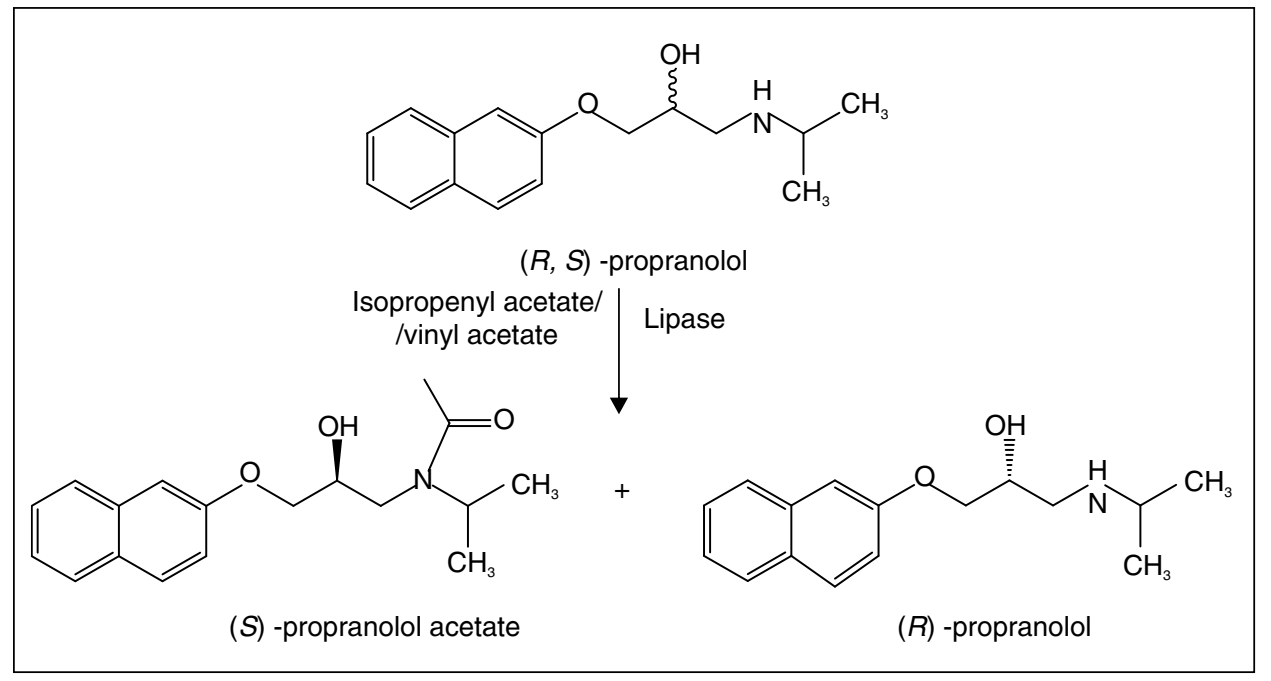

Figure 2. Enantioselective acetylation of racemic propranolol with the use of lipase as biocatalyst.

\section{Chemical acetylation of (R,S)-propranolol}

The $(R, S)$-propranolol was acetylated according to the reported methodology $(10,12)$ with few modifications. Briefly, $(R, S)$-propranolol $(0.02 \mathrm{~g} ; 0.07 \mathrm{mmol})$ was refluxed with dichloromethane $(20 \mathrm{~mL})$, and acetyl chloride ( $8 \mu \mathrm{l} ; 0.076 \mathrm{mmol}$ ) was slowly added. Further, the reaction mixture was incubated at $30^{\circ} \mathrm{C}$ for $2 \mathrm{~h}$ and successively washed with equal volumes of brine and saturated aqueous sodium bicarbonate. Next, the organic layer was collected and evaporated under vacuum to afford propranolol acetate. Finally, the resulting derivate of propranolol was used as a standard to establish an optimal chromatographic method for quantitative and qualitative determination of racemic propranolol and its acetylated form.

\section{Enantioselective acetylation of $(\mathrm{R}, \mathrm{S})$-propranolol}

Enantioselective biotransformation of $(R, S)$-propranolol using vinyl acetate or isopropenyl acetate as the acetylating agent resulted in the production of $N$-acetyl-propranolol (Figure 2). Because the solubility of racemic propranolol is low in most organic solvents, the reaction was performed in $10 \mathrm{~mL}$ of the reaction medium. The reaction was performed with different solvents, e.g. acetonitrile, dichloromethane, diisopropyl ether, tert-butyl-methyl ether, chloroform, tetrahydrofuran, toluene, and racemic propranolol $(3.0 \mathrm{mg})$, and vinyl acetate or isopropenyl acetate $(2-10 \mu \mathrm{L})$ as the acetyl donor. The reaction was started by the addition of $10 \mathrm{mg}$ of lipase in native form. The reaction mixture was shaken at $250 \mathrm{rpm}$ at $\mathrm{t}=37^{\circ} \mathrm{C}$. The process of enantioselective acetylation was monitored using chiral stationary phases and a UPLC-MS/MS system. The samples were withdrawn at previously established time points $(24,48,72$, and $96 \mathrm{~h})$ and then evaporated and re-dissolved in pure acetonitrile, filtered, and injected into the UPLC-MS/MS system.

\section{Results and discussion}

\section{Effect of acetylating agent}

The effect of the acetylating agent was investigated in order to optimise the reaction conditions. The vinyl acetate as well as isopropenyl acetate were tested at different concentrations. The biocatalytic system consisted of toluene as a reaction medium, racemic propranolol $(3.0 \mathrm{mg}$ ), lipase from Candida rugosa OF (10 mg), and an appropriate acetylating agent. The reaction was carried out at ambient temperature for $96 \mathrm{~h}$. As shown in Figure 3, the best reaction parameters were achieved by using isopropenyl acetate in the concentration $0.018 \mathrm{mmol}(2 \mu \mathrm{L})$. Additionally, the use of vinyl acetate resulted in obtaining many by-products, which could not be identified; therefore, it was decided to use isopropenyl acetate in further steps of study optimisation in case of the solvent and lipase screening.

\section{Effect of organic solvent}

The effect of organic solvent on the properties of enzymatic kinetic resolution of racemic propranolol was investigated. It was decided to perform enantioselective biocatalysis in $10 \mathrm{~mL}$ of reaction medium due to the low solubility of racemic propranolol. Additionally, all of the tested solvents were organic because of the characteristics of enantioselective biotransformation, which relied on 


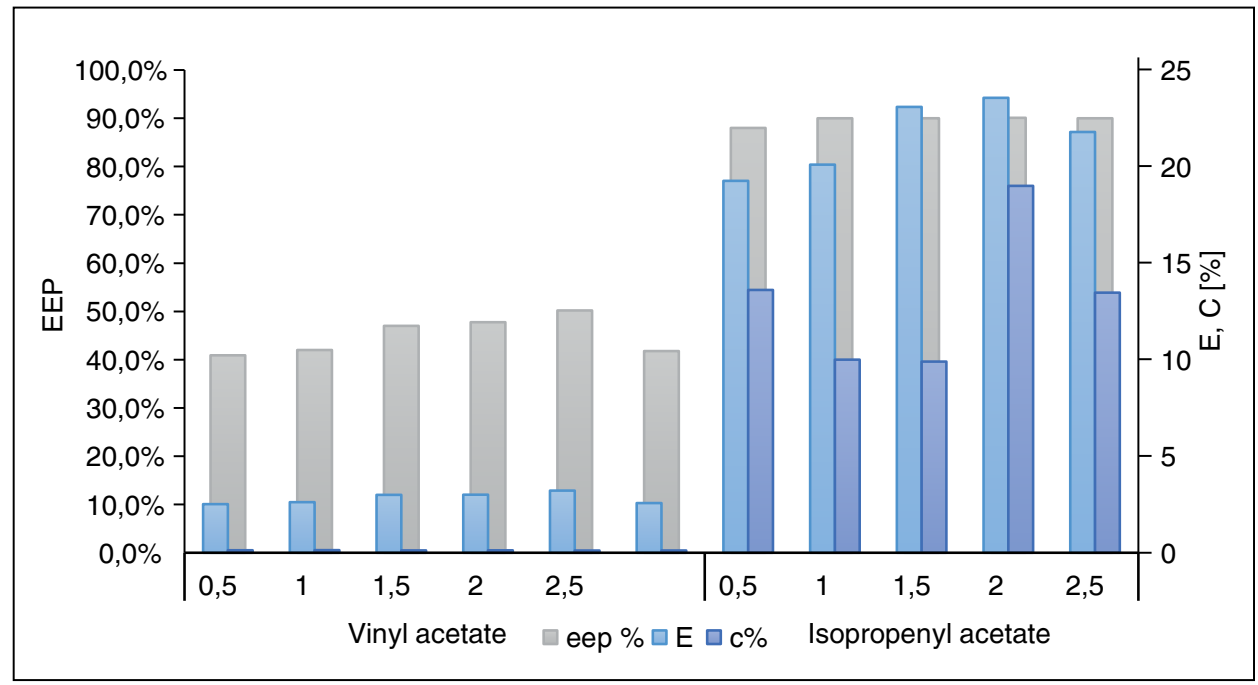

Figure 3. Enzymatic parameters including enantioselectivity (E), enantiomeric excess of product (eep), and conversion (c) of different acetylating agents screened for the enantioselective acetylation of racemic propranolol. Reaction conditions: Candida rugosa lipase (OF) (10 mg), $(R, S)$-propranolol $(3 \mathrm{mg})$, toluene $(10 \mathrm{~mL})$, vinyl acetate or isopropenyl acetate (0.5-2.5 $\mu \mathrm{L})$, time $-96 \mathrm{~h}$, temperature $-37^{\circ} \mathrm{C}, \mathrm{RPM}-250$.

Table 1. Enzymatic parameters including enantioselectivity $(E)$, enantiomeric excesses of both substrate $\left(e_{s}\right)$ and product $\left(\mathrm{ee}_{\mathrm{p}}\right)$, and conversion (c) of enantioselective acetylation of $(R, S)$-propranolol with the use of lipase from Candida rugosa OF after $96 \mathrm{~h}$ of incubation.

\begin{tabular}{lcccc}
\hline Organic solvent & $\mathbf{E}$ & $\mathbf{e e}_{\mathbf{s}}[\%]$ & $\mathbf{e e}_{\mathbf{p}}$ [\%] & $\mathbf{c}[\%]$ \\
\hline Acetonitrile & 2.07 & 0.62 & 34.54 & 1.76 \\
Chloroform & 1.31 & 3.62 & 11.69 & 23.64 \\
diisopropyl ether & 7.36 & 13.95 & 73.07 & 16.03 \\
t-butyl-methyl ether & 4.96 & 0.76 & 66.23 & 1.14 \\
Tetrahydrofuran & 1.24 & 2.34 & 9.70 & 19.44 \\
Toluene & 23.56 & 21.38 & 90.06 & 19.19 \\
\hline
\end{tabular}

reaction of transesterification. The reaction mixture in that step of the study consisted of a different reaction medium, i.e. acetonitrile, chloroform, tetrahydrofuran, $t$-butyl-methyl ether, diisopropyl ether or toluene, lipase from Candida rugosa OF $(10 \mathrm{mg})$, isopropenyl acetate $(0.018 \mathrm{mmol})$ as an acetylating agent, and racemic propranolol (3.0 mg).

The catalytic properties of an enzyme are mainly affected by the hydrophobicity of the solvent. Therefore, the optimal choice of solvent as reaction medium is one of the most important parts of optimising reaction conditions for achievement of the best value of enantioselectivity. Among all tested solvents, only toluene proved to be suitable for performing the enantioselective acetylation of racemic propranolol, as shown in Table 1. Even though the solubility of propranolol is higher in other solvents than toluene, these solvents are not suitable for the kinetic resolution of $(R, S)$-propranolol because the enantiomeric excess of product and enantioselectivity are low. Additionally, the use of toluene made it possible to obtain a high value of enantioselectivity, which was above 20 . Based on abovementioned results, toluene as a reaction medium was selected for further investigation of lipase screening.

\section{Screening of lipases}

Commercially available lipases from Aspergilus niger, Burkholderia cepacia (BCL, SL), Candida antarctica (CAL$\mathrm{BY}$ ), Candida rugosa (MY, OF), Alcaigenes fecalis (QLM), Alcaigenes sp. (PL), and Pseudomonas strutzeri (TL) were tested for their catalytic properties in the enantioselective acetylation of racemic propranolol with isopropenyl acetate as an acetylating agent, and using toluene as the reaction medium. As shown in Table 2, among all tested enzymes, the most satisfactory results were obtained for Candida rugosa lipases (OF, MY). However, it should be emphasised that the enantiomeric ratio and enantiomeric excess of product were higher in reactions using lipase 
Table 2. Enzymatic parameters including enantioselectivity $(E)$, enantiomeric excesses of both substrate (ee $)_{s}$ and product $\left(e_{\mathrm{p}}\right)$, and conversion (c) of enantioselective acetylation of $(R, S)$-propranolol with the use of various native lipases in toluene after $96 \mathrm{~h}$ of incubation.

\begin{tabular}{lcccc}
\hline Lipase & E & ees \% & eep \% & c \% \\
\hline Alcaigenes fecalis (QLM) & 1.12 & 1.71 & 5.07 & 25.17 \\
Alcaigenes sp. (PL) & 1.20 & 5.24 & 6.93 & 43.04 \\
Aspergilus niger & 1.24 & 8.34 & 7.06 & 54.16 \\
Burkholderia cepacia (BCL) & 3.22 & 6.55 & 50.23 & 11.53 \\
Burkholderia cepacia (SL) & 2.57 & 5.58 & 41.80 & 11.77 \\
Candida antarctica (CALBY) & 3.01 & 6.86 & 47.53 & 12.61 \\
Candida rugosa (MY) & $\mathbf{2 1 . 8 0}$ & 14.00 & 90.00 & $\mathbf{1 3 . 4 6}$ \\
Candida rugosa (OF) & $\mathbf{2 3 . 5 6}$ & $\mathbf{2 1 . 3 8}$ & 90.06 & $\mathbf{1 9 . 1 9}$ \\
Pseudomonas strutzeri (TL) & 2.78 & 2.09 & 46.25 & 4.32 \\
\hline
\end{tabular}

OF than using lipase MY. The other lipases tested did not provide any promising results, and their use resulted in significantly lower values of enantioselectivity and enantiomeric excesses of both substrate and product.
The project was supported by a research grant from the Faculty of Pharmacy, Collegium Medicum in Bydgoszcz, Nicolaus Copernicus University in Toruń, no. MN-SDF-6/WF/2017.

\section{Conclusions}

The reported results confirm that nine commercially available lipases have different effects on the kinetic resolution of $(R, S)$-propranolol. The type of lipase, the concentration of the acetylating agent, and the reaction medium have significant impacts on the efficiency of the catalyst system. The most effective conversion and the highest enantioselectivity were obtained using lipase from Candida rugosa (OF). Among all tested reaction mediums, toluene gave the highest enantioselectivity. The crucial point is that many different reaction factors influence the effectiveness of the enantioselective acetylation of $\beta$-blockers. Finally, the reaction mixture composed of Candida rugosa OF lipase, toluene as the reaction medium, and isopropenyl acetate as the acetylating agent made it possible to obtain enantiomerically pure $(S)$-propranolol acetate with ee $e_{p}=90.1 \%$. However, it is necessary to optimise the methods for the kinetic resolution of chiral drugs to obtain enantiomerically pure products, and thus it is not possible to find a universal catalytic system for all syntheses because each enzymatic process requires an individual approach.

\section{Acknowledgments}

The authors wish to express their sincere thanks to Meito Sangyo Co. (Japan) for the supply of lipases OF and MY from Candida rugosa, lipase QLM from Alcaigenes fecalis, lipase PL from Alcaigenes sp., lipase TL from Pseudomonas stutzeri, and lipase SL from Burkholderia cepacia.

\section{References}

1. Narina SV, Sudalai A. Enantioselective synthesis of (S)-timolol via kinetic resolution of terminal epoxides dihydroxylation of allylamines ( $\mathrm{vol} 63$, pg 3026, 2007). Tetrahedron. 2007; 63(29): 6938.

2. Carlberg Bo, Samuelsson O, Lindholm LH. Atenolol in hypertension: is it a wise choice? Lancet. 2004; 364(9446): 1684-1689, doi: 10.1016/S0140-6736(04)17355-8, indexed in Pubmed: 15530629.

3. Lund I, Bøckmann P, Jacobsen E. Highly enantioselective CALB-catalyzed kinetic resolution of building blocks for -blocker atenolol. Tetrahedron. 2016; 72(46): 7288-7292, doi: 10.1016/j.tet.2016.02.018.

4 Barbosa O, Ariza C, Ortiz C et al. Kinetic resolution of (R/S)-propranolol (1-isopropylamino-3-(1-naphtoxy)-2-propanolol) catalyzed by immobilized preparations of Candida antarctica lipase $B(C A L-B)$. N Biotechnol. 2010; 27(6): 844-850, doi: 10.1016/j.nbt.2010.07.015, indexed in Pubmed: 20667519

5. Zelaszczyk D, Kieć-Kononowicz K. Biocatalytic approaches to optically active beta-blockers. Curr Med Chem. 2007; 14(1): 53-65, doi: 10.2174/092986707779313480, indexed in Pubmed: 17266567.

6. Escorcia A, Daza M, Doerr M. Computational study of the enantioselectivity of the O-acetylation of $(R, S)$-propranolol catalyzed by Candida antarctica lipase B. J Mol Catal B-Enzym. 2014; 108: 21-31, doi: 10.1016/j.molcatb.2014.06.010.

7. Dwivedee B, Ghosh S, Bhaumik J, et al. Lipase-catalyzed green synthesis of enantiopure atenolol. RSC Adv. 2015; 5(21): 15850-15860, doi: 10.1039/c4ra16365f.

8. Escorcia A, Molina D, Daza M, et al. Acetylation of (R,S)-propranolol catalyzed by Candida antarctica lipase B: An experimental and computational study. J Mol Catal B-Enzym. 2013; 98: 21-29, doi: 10.1016/j. molcatb.2013.09.019.

9. Chiou TW, Chang CC, Lai CT, et al. Kinetic resolution of propranolol by a lipase-catalyzed N-acetylation. Bioorg Med Chem Lett. 1997; 7(4): 433-436, doi: 10.1016/s0960-894x(97)00028-0.

10. Sikora A, Chełminiak-Dudkiewicz D, Ziegler-Borowska M, et al. Enantioseparation of (RS)-atenolol with the use of lipases immobilized onto new-synthesized magnetic nanoparticles. Tetrahedron-Asymmetr. 2017; 28(2): 374-380, doi: 10.1016/j.tetasy.2017.01.012.

11. Siódmiak T, Mangelings D, Vander Heyden $Y$, et al. High enantioselective Novozym 435-catalyzed esterification of (R,S)-flurbiprofen monitored with a chiral stationary phase. Appl Biochem Biotechnol. 2015; 175(5): 2769-2785, doi: 10.1007/s12010-014-1455-4, indexed in Pubmed: 25561056.

12. Sikora A, Chełminiak-Dudkiewicz D, Siódmiak T, et al. Enantioselective acetylation of ( $\mathrm{R}, \mathrm{S}$ )-atenolol: The use of Candida rugosa lipases immobilized onto magnetic chitosan nanoparticles in enzyme-catalyzed biotransformation. J Mol Catal B-Enzym. 2016; 134: 43-50, doi: 10.1016/j.molcatb.2016.09.017. 